\title{
Subtle mutations in the SMN1 gene in Chinese patients with SMA: p.Arg288Met mutation causing SMN1 transcript exclusion of exon7
}

\author{
Qu Yu-jin' ${ }^{1}$ Du Juan ${ }^{1}$, Li Er-zhenn ${ }^{2}$, Bai Jin-li', Jin Yu-wei ${ }^{1}$, Wang Hong ${ }^{1}$ and Song Fang ${ }^{1 *}$
}

\begin{abstract}
Background: Proximal spinal muscular atrophy (SMA) is a common neuromuscular disorder resulting in death during childhood. Around $81 \sim 95 \%$ of SMA cases are a result of homozygous deletions of survival motor neuron gene 1 (SMN1) gene or gene conversions from SMN1 to SMN2. Less than 5\% of cases showed rare subtle mutations in SMN1. Our aim was to identify subtle mutations in Chinese SMA patients carrying a single SMN1 copy.

Methods: We examined 14 patients from 13 unrelated families. Multiplex ligation-dependent probe amplification analysis was carried out to determine the copy numbers of SMN1 and SMN2. Reverse transcription polymerase chain reaction (RT-PCR) and clone sequencing were used to detect subtle mutations in SMN1. SMN transcript levels were determined using quantitative RT-PCR.

Results: Six subtle mutations (p.Ser8LysfsX23, p.Glu134Lys, p.Leu228X, p.Ser230Leu, p.Tyr277Cys, and p.Arg288Met) were identified in 12 patients. The p.Tyr277Cys mutation has not been reported previously. The p.Ser8LysfsX23, p.Leu228X, and p.Tyr277Cys mutations have only been reported in Chinese SMA patients and the first two mutations seem to be the common ones. Levels of full length SMN1 (fl-SMN1) transcripts were very low in patients carrying p.Ser8LysfsX23, p.Leu228X or p.Arg288Met compared with healthy carriers. In patients carrying p.Glu134Lys or p.Ser230Leu, levels of fl-SMN1 transcripts were reduced but not significant. The SMN1 transcript almost skipped exon 7 entirely in patients with the p.Arg288Met mutation.

Conclusions: Our study reveals a distinct spectrum of subtle mutations in SMN1 of Chinese SMA patients from that of other ethnicities. The p.Arg288Met missense mutation possibly influences the correct splicing of exon 7 in SMN1. Mutation analysis of the SMN1 gene in Chinese patients may contribute to the identification of potential ethnic differences and enrich the SMN1 subtle mutation database.
\end{abstract}

Keywords: Spinal muscular atrophy, Survival motor neuron gene-1, Subtle mutation, Transcript

\section{Background}

Proximal spinal muscular atrophy (SMA) is a common infantile neuromuscular disease with an incidence of 1 in $6000 \sim 10,000$ among newborns [1]. It is characterized by the destruction of alpha motor neurons in anterior horn cells of the spinal cord. This leads to progressive symmetrical limb and trunk muscle weakness, along with atrophy [2]. This disease can be categorized into four clinical types

\footnotetext{
* Correspondence: songf_558@263.net

'Department of Medical Genetics, Capital Institute of Pediatrics, Beijing, China

Full list of author information is available at the end of the article
}

(SMA I-IV) based on age at onset and maximum attained motor functions [3].

The SMA-determining gene, survival motor neuron $(S M N)$, is located at chromosome 5q11.2-13.3 [4], with two almost identical copies: telomeric SMN1 (MIM\#600354; GenBank: NM_000344) and centromeric SMN2 (MIM\#601627; GenBank: NM_022875). SMN1 and SMN2 sequences are highly homologous with only five nucleotide differences, one in intron 6 , one in exon 7, two in intron 7, and one in exon 8 [4]. Although these differences do not alter the encoded amino acids, SMN1 produces mainly full-length $S M N$ transcripts. However, $90 \%$ of the transcripts produced by SMN2 exclude exon

\section{Ciomed Central}


7 owing to a conversion $(\mathrm{C} \rightarrow \mathrm{T})$ at position 840 in exon 7 [5]. Mutations in SMN1 result in the SMA phenotype, whereas SMN2 copy numbers determine the severity of SMA.

The majority of SMA patients have been found to have a homozygous deletion in exon 7 of SMN1. Some SMA cases are caused by compound mutations, with a SMN1 deletion on one allele and a subtle mutation on the other. Since the first several mutations were identified at 1995 by Lefebvre et al. [4] and Bussaglia et al. [6], More than 60 subtle mutations of SMN1 gene have been continuously identified worldwide [7-24]. Although mutations are distributed along the entire coding sequence of $S M N 1$, the majority are located in exons 3 and 6 $(47.5 \%)$. Tsai et al. reported the first subtle mutation in SMN1 of Chinese SMA patients in 2001 [18]. To date, eight subtle mutations have been successfully identified in Chinese SMA patients [18-23]. In this article, our aim was to identify subtle mutations in SMN1 of Chinese SMA patients and detect the SMN1 transcript levels of these patients based on the quantitative RT-PCR.

\section{Methods}

\section{Patients and materials}

Fourteen SMA patients and their parents, from 13 unrelated families, were enrolled in this study. All patients met the diagnostic criteria of proximal SMA, with their clinical data provided in Table 1 . Of these cases, three were diagnosed as type I SMA, nine were type II SMA, and two were type III SMA. Case 9 was the younger sister of case 8. Genomic DNA and total RNA from these individuals were isolated from peripheral venous blood using phenol-chloroform extraction and an RNeasy Kit (Qiagen, Germany), respectively. Samples of the parents of cases 1 and 7 were not available. This study was approved by the Ethics Committee of the Capital Institute of Pediatrics, and informed consent was obtained from all subjects.

\section{Analysis of subtle mutations in SMN1}

Cloning and sequencing of reverse transcription polymerase chain reaction (RT-PCR) amplicons was carried out to analyze subtle mutations. First-strand cDNA synthesis was performed with $0.5 \mu \mathrm{g}$ of total RNA, random primers, and M-MLV Reverse Transcriptase (Invitrogen, USA) in accordance with the manufacturer's instructions. Specific PCR primers (SMN575 [13] and $541 C 1120$ [4]), were used to amplify the SMN gene (exons 1-8) using LA Taq polymerase (TAKARA, Japan) and cDNA template. Thermal cycling conditions involved an initial denaturation step for $5 \mathrm{~min}$ at $94^{\circ} \mathrm{C}$, followed by 30 cycles of $45 \mathrm{~s}$ at $94^{\circ} \mathrm{C}, 50 \mathrm{~s}$ at $60^{\circ} \mathrm{C}$, and $60 \mathrm{~s}$ at $72^{\circ} \mathrm{C}$, with a final extension step at $72^{\circ} \mathrm{C}$ for $10 \mathrm{~min}$. Amplicons were subcloned into the pGEM-T
Easy cloning vector (Promega, USA) according to the supplier's protocol. SMN1and SMN2 subclones were differentiated using restriction enzymes (DraI and DdeI) [25]. Around 5 8 SMN1 and 2 3 SMN2 clones for each case were sequenced. Mutations were further confirmed by direct sequencing of the amplified products from $S M N$ genomic DNA samples.

\section{Multiplex ligation-dependent probe amplification (MLPA) analysis}

MLPA analysis was performed to detect copy numbers of SMN1 and SMN2 in all cases using a SALSA MLPA kit (P021-A1; MRC-Holland, Amsterdam, The Netherlands) according to the manufacturer's recommendations. This SALSA kit contained 16 probes specific for the SMA critical region (5q12.2-q13.3). Among these, two specific probes for the $\mathrm{C} \rightarrow \mathrm{T}$ transition in exon 7 (C for SMN1 and $\mathrm{T}$ for $S M N 2$ ) and two specific probes were for the $\mathrm{G} \rightarrow \mathrm{A}$ transition in exon 8 ( $\mathrm{G}$ for SMN1 and A for $S M N 2)$ were included. In addition, the SALSA kit contained 21 control probes mapping to other autosomes. After MLPA treatment, products were run on the ABI 3730 automatic sequencing system (Applied Bio-Systems, USA). Four healthy individuals carrying two SMN1 copies were the normal controls, with eight carriers (parents of the patients with a homozygous SMN1 deletion) as the single copy SMN1 reference. For each sample, raw data [relative peak area (RPA)] were analyzed and compared with normal controls using Gene marker version 1.75 software. This software is able to calculate the RPA for each probe and to compare RPAs with those derived from normal controls. All samples were analyzed at least twice. A ratio with normal controls in the range 0.7-1.3 indicated a normal copy number (two copies), a ratio less than 0.7 indicated one copy, a ratio between $1.3-1.6$ was indicative of three copies, and a ratio equal to 0 indicated zero copy.

\section{Analysis of the novel p.Tyr277Cys mutation}

The allele-specific primer, Y277CR (5/-CTG AGT GAT TAC TTA CCA TAC-3'), was designed to identify only the mutant allelic sequence, and was coupled with the upstream primer SMNE6F [12]. This was applied in an allele-specific PCR (AS-PCR) to screen for the p.Tyr277Cys mutation in 150 control individuals. Simultaneously, alignment analysis of SMN proteins from six different species was performed using Clustal X version 1.8 to analyze levels of conservation for tyrosine 277 .

\section{Restriction endonuclease digestion of SMN transcripts}

Total RNA $(0.5 \mu \mathrm{g})$ was isolated from the peripheral blood of patients and controls, and used to synthesize first-strand cDNA as described earlier. The cDNAs were amplified using primers SMN575 [13] and 541C1120 [4] 
Table 1 Genotype and phenotype in patients with a subtle mutation of SMN1 gene

\begin{tabular}{|c|c|c|c|c|c|c|c|c|c|c|c|c|c|c|c|}
\hline \multirow{2}{*}{$\begin{array}{l}\text { Family } \\
\text { No. }\end{array}$} & \multirow{2}{*}{$\begin{array}{l}\text { Case } \\
\text { No. }\end{array}$} & \multirow[t]{2}{*}{ PhenoType } & \multirow[t]{2}{*}{ Gender } & \multirow{2}{*}{$\begin{array}{l}\text { Age at last } \\
\text { examination }\end{array}$} & \multirow{2}{*}{$\begin{array}{l}\text { Age of } \\
\text { onset }\end{array}$} & \multicolumn{3}{|c|}{ Attained motor function } & \multicolumn{2}{|c|}{ SMN1 genotype } & \multirow{2}{*}{$\begin{array}{c}\text { Point } \\
\text { Mutation } \\
\text { location }\end{array}$} & \multirow{2}{*}{$\begin{array}{l}\text { SMN2 } \\
\text { copies }\end{array}$} & \multirow{2}{*}{$\begin{array}{l}\text { FI-SMN2 } \\
\text { transcript }\end{array}$} & \multirow{2}{*}{$\begin{array}{c}F I-S M N \\
\text { transcript }\end{array}$} & \multirow{2}{*}{$\begin{array}{l}\text { Parental } \\
\text { origin }\end{array}$} \\
\hline & & & & & & $\begin{array}{l}\text { Head } \\
\text { control }\end{array}$ & $\begin{array}{c}\text { Sit } \\
\text { unsupported }\end{array}$ & $\begin{array}{c}\text { Walk } \\
\text { independently }\end{array}$ & Allele1 & Allele2 & & & & & \\
\hline 1 & 1 & । & M & $3 y 6 m$ & $4 \mathrm{~m}$ & + & - & - & Deletion & p.Ser8LysfsX23 & Exon 1 & 2 & - & - & ND \\
\hline 2 & 2 & $\|$ & M & $1 y 7 m$ & $11 \mathrm{~m}$ & + & + & - & Deletion & p.Ser8LysfsX23 & Exon 1 & 2 & $6.33 \pm 1.72$ & $6.76 \pm 1.94$ & Paternal \\
\hline 3 & 3 & $\|$ & M & $6 y 8 m$ & $1 y 2 m$ & + & + & - & Conversion & p.Ser8LysfsX23 & Exon 1 & 3 & $20.36 \pm 11.09$ & $21.01 \pm 10.4$ & Paternal \\
\hline 4 & 4 & $\|$ & M & $5 y$ & $11 \mathrm{~m}$ & + & + & - & Deletion & p.Glu134Lys & Exon 3 & 2 & $7.73 \pm 5.44$ & $12.46 \pm 5.47$ & Paternal \\
\hline 5 & 5 & $\|$ & $\mathrm{F}$ & $2 \mathrm{y} 6 \mathrm{~m}$ & $1 \mathrm{y} 1 \mathrm{~m}$ & + & + & - & Deletion & p.Glu134Lys & Exon 3 & 2 & $6.41 \pm 5.78$ & $11.21 \pm 3.45$ & Paternal \\
\hline 6 & 6 & । & $\mathrm{F}$ & $2 y 3 m$ & $4 \mathrm{~m}$ & + & - & - & Deletion & p.Leu228X & Exon 5 & 2 & $9.24 \pm 6.6$ & $10.277 \pm 6.8$ & Maternal \\
\hline 7 & 7 & $\|$ & M & $4 y 1 m$ & $8 \mathrm{~m}$ & + & + & - & Conversion & p.Leu228X & Exon 5 & 3 & - & - & ND \\
\hline \multirow[t]{2}{*}{8} & 8 & $\|$ & M & $14 y$ & $10 \mathrm{~m}$ & + & + & - & Deletion & p.Ser230Leu & Exon 5 & 2 & $8.04 \pm 7.27$ & $12.22 \pm 7.07$ & Paternal \\
\hline & 9 & III & $\mathrm{F}$ & $9 y 8 m$ & $2 y$ & + & + & $+\dagger$ & Deletion & p.Ser230Leu & Exon 5 & 2 & $9.35 \pm 5.74$ & $14.83 \pm 4.45$ & Paternal \\
\hline 9 & 10 & $\|$ & M & $6 y 4 m$ & $1 y$ & + & + & - & Deletion & p.Tyr277Cys§ & Exon 6 & 2 & $4.53 \pm 3.47$ & $6.54 \pm 4.33$ & Maternal \\
\hline 10 & $11 \#$ & I & $\mathrm{F}$ & $2 \mathrm{y} 10 \mathrm{~m}$ & $5 \mathrm{~m}$ & + & - & - & Conversion & p.Arg288Met & Exon 7 & 3 & $13.1 \pm 10.4$ & $13.71 \pm 10.6$ & Paternal \\
\hline 11 & $12 \#$ & $\|$ & $\mathrm{F}$ & $4 y 4 m$ & $1 \mathrm{y} 6 \mathrm{~m}$ & + & + & - & Conversion & p.Arg288Met & Exon 7 & 3 & $9.46 \pm 7.84$ & $12.46 \pm 8.01$ & Paternal \\
\hline 12 & 13 & $\|$ & M & $5 y$ & $10 \mathrm{~m}$ & + & + & - & Deletion & - & - & 2 & $8.02 \pm 1.97$ & $9.86 \pm 2.01$ & ND \\
\hline 13 & 14 & III & M & $15 y$ & $1 \mathrm{y} 5 \mathrm{~m}$ & + & + & $+\dagger$ & Conversion & - & - & 3 & $21.67 \pm 11.0$ & $25.17 \pm 11.8$ & ND \\
\hline
\end{tabular}

Conversion means deletion of $S M N 1$ owing to conversion of $S M N 1$ sequences to $S M N 2$ with the copy number of SMN2 increasing. ND, not detected. §Novel mutation; \#,these patients had been reported [22]; $\dagger$, Walk in waddling gait; case 8 and case 9 are siblings. 
Table 2 Information of Primers and Probes Used for qRT-PCR

\begin{tabular}{llll}
\hline Fragment & Primers & Probe & Amplify length \\
\hline SMN1 & SMN_mgb-F: 5'TGGTACATGAGTGGCTATCATACTG3' & 5'FAM-ATGGGTTTCAGAA-MGB-NFQ & 75 bp \\
& SMN_mgb-R: 5' GTGAGCACCTTCCTTCTTITT3' & & \\
SMN2 & SMN_mgb-F: 5'TGGTACATGAGTGGCTATCATACTG3' & 5/FAM-ATGGGTTITAGAA-MGB-NFQ & 75 bp \\
& SMN_mgb-R: 5' GTGAGCACCTTCCTTCTTTTT3' & & 73 bp \\
GAPDH[26] & GAPDH_abs-F:5'GGGTGTGAACCATGAGAAGTATGA-3' & 5/FAM-CAAGATCATCAGCAATGC-NFQ3' & \\
& GAPDH_abs-R: 5'CTAAGCAGTTGGTGGTGCAGG-3' & & \\
\hline
\end{tabular}

The bases $\mathbf{C}$ and $\mathbf{T}$ in bold are specific for SMN1 and SMN2, respectively. The primers and probe sequences of GAPDH are described by Tiziano FD et al. [26].

in a $50-\mu$ l reaction with a $60^{\circ} \mathrm{C}$ annealing temperature for 30 cycles as the section of "Analysis of subtle mutations in SMN1 "described. In general, SMN transcripts yielded three products, full-length SMN1 (fl-SMN1, 1259 bp), full-length SMN2 (fl-SMN2, $1259 \mathrm{bp)}$ and $S M N 2$ isoform lacking exon 7 ( $\triangle 7-S M N 2,1205$ bp). The SMN1 transcripts could be distinguished from SMN2 transcripts by digestion with the restriction enzyme DdeI. Following digestion, there was a 1259-bp fragment corresponding to fl-SMN1, a 1136-bp fragment corresponding to fl-SMN2, a 1082-bp fragment indicative of $\triangle 7-S M N 2$ and a 123-bp fragment from SMN2. The transcripts and their products after DdeI digestion were separated on a $6 \%$ polyacrylamide gel at 500 volts for $2 \mathrm{~h}$. Gels were stained with silver stain.

\section{Quantitative RT-PCR (qRT-PCR)}

Three plasmids (fl-SMN1, fl-SMN2 and GAPDH) were constructed as external standards. Amplified SMN1 and SMN2 were obtained using normal control cDNA and primers SMN-F (5'-GCT GAT GCT TTG GGA AGT ATG TTA-3') and SMN-R (5/-TCA ACT GCC TCA CCA CCG TGC TGG-3/), specific for exons 6 and 8, respectively. The primer pair for amplification of GAPDH

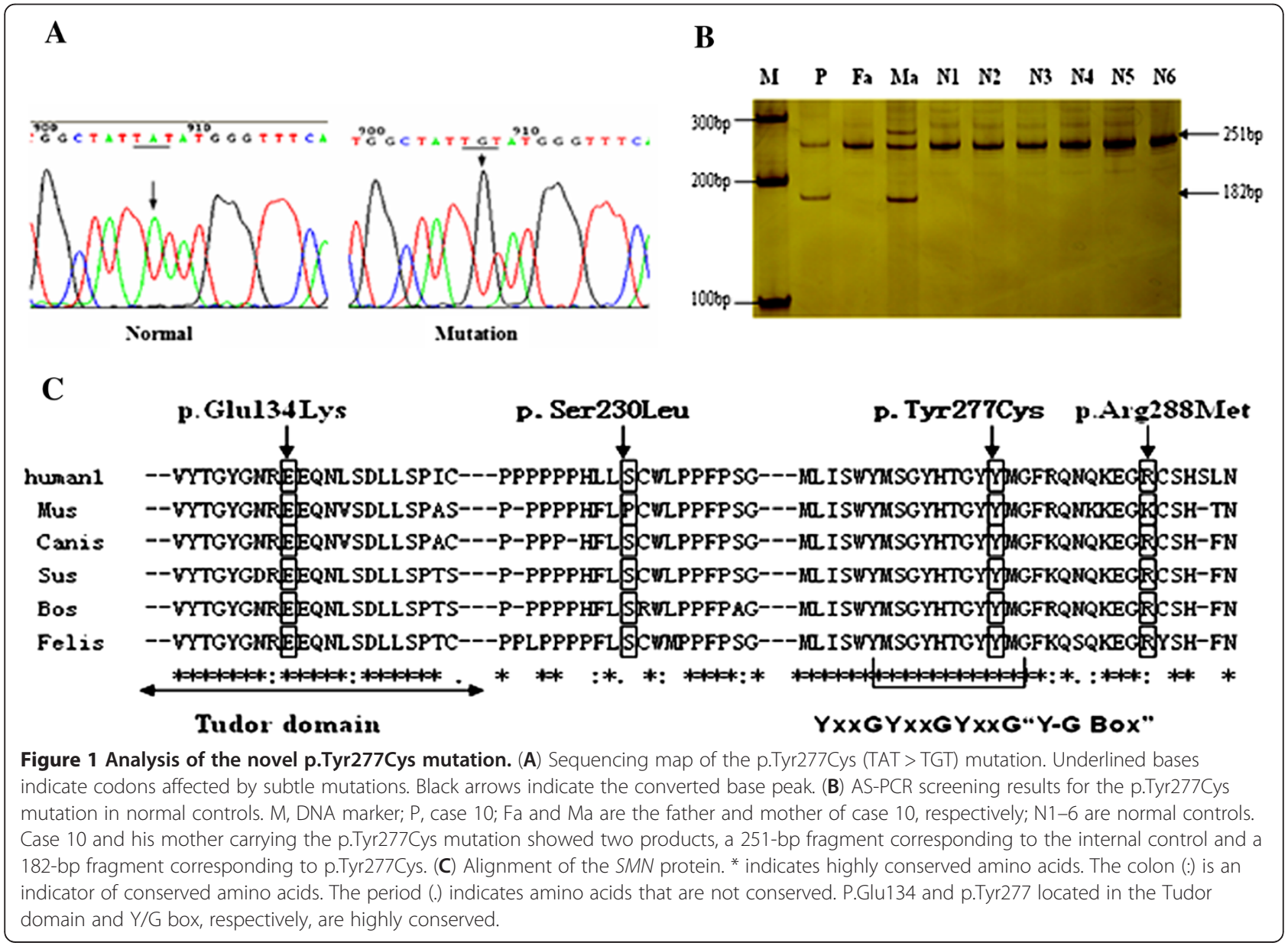


was GAPDH_exst-F and GAPDH_exst-R, as previously described [26]. Amplicons for SMN1/SMN2 (395 bp) and GAPDH (133 bp) were cloned into the pGEM-T Easy cloning vector (Promega, USA). Cloned inserts were all verified by sequencing. Plasmid DNA was extracted and quantified by absorbance using a NanoDrop 2000 (Thermo, USA). Based on plasmid length and concentration, the copy number of each plasmid can be calculated. These plasmids were serially diluted across a range $\left(10^{3}-10^{8}\right.$ copies) and used as external standards to construct the standard curve.

A qPCR assay to quantify $S M N$ transcripts was conducted as described by Tiziano et al. [26]. The primers and MGB-probes were designed using Primer Express v1.5 software (Applied Biosystems, USA), with sequences provided in Table 2. The fl-SMN1 and fl-SMN2 transcripts were amplified using the same primer pair (SMN_mgb-F and SMN_mgb-R). Full-length transcripts of the two genes were distinguished by two different Taqman MGB probes on the basis of the $\mathrm{C} \rightarrow \mathrm{T}$ transition located in exon 7. For GAPDH, the primers (GAPDH_abs-F and GAPDH_abs-R) and MGB probe sequence were the same as those described in Tiziano et al. [26] described. All reactions $(20 \mu \mathrm{l})$ were carried out using a 7500 Real-Time PCR System (Applied Biosystems, USA) and contained $2 \times$ GoldStar TaqMan Mixture (KANGWEI, China), $20 \mathrm{ng}$ of cDNA, $0.4 \mu \mathrm{l}$ of each primer $(10 \mathrm{pmol} /$ $\mu \mathrm{l})$, and $4 \mathrm{pmol}$ of the SMN1, SMN2 or GAPDH probe. The thermal cycling conditions involved $2 \mathrm{~min}$ at $50^{\circ} \mathrm{C}$ then $10 \mathrm{~min}$ at $95^{\circ} \mathrm{C}$, followed by 40 cycles of $15 \mathrm{~s}$ at $95^{\circ} \mathrm{C}$ and $1 \mathrm{~min}$ at $60^{\circ} \mathrm{C}$. Each sample was assayed in duplicate and repeated at least twice. Evaluation of data was performed using 7500 Software SDS version 1.4.

\section{Statistical analysis}

Transcript levels for fl-SMN1, fl-SMN2 and GAPDH were expressed as copies per nanogram of total RNA. Expression levels of fl-SMN1, fl-SMN2, and fl-SMN were normalized to $G A P D H$. Statistical analysis was carried out using SPSS 19.0. A parametric test (t-test) was used to compare the transcript levels between normal controls and carriers, as well as between carriers and patients. Correlation between SMN2 gene copy numbers and fl-SMN2 transcript levels were analyzed by a gerneral linear model(one-way ANOVA). A $p$-value less than 0.05 was regarded as significant.

\section{Results}

\section{SMN1 and SMN2 copy numbers}

The numbers of SMN1 and SMN2 copies in the 14 patients analyzed by MLPA are presented in Table 1 . All patients carried only one copy of SMN1. Patients 3, 7, 11,12 , and 14 carried three copies of SMN2. The remaining patients had two copies of SMN2.

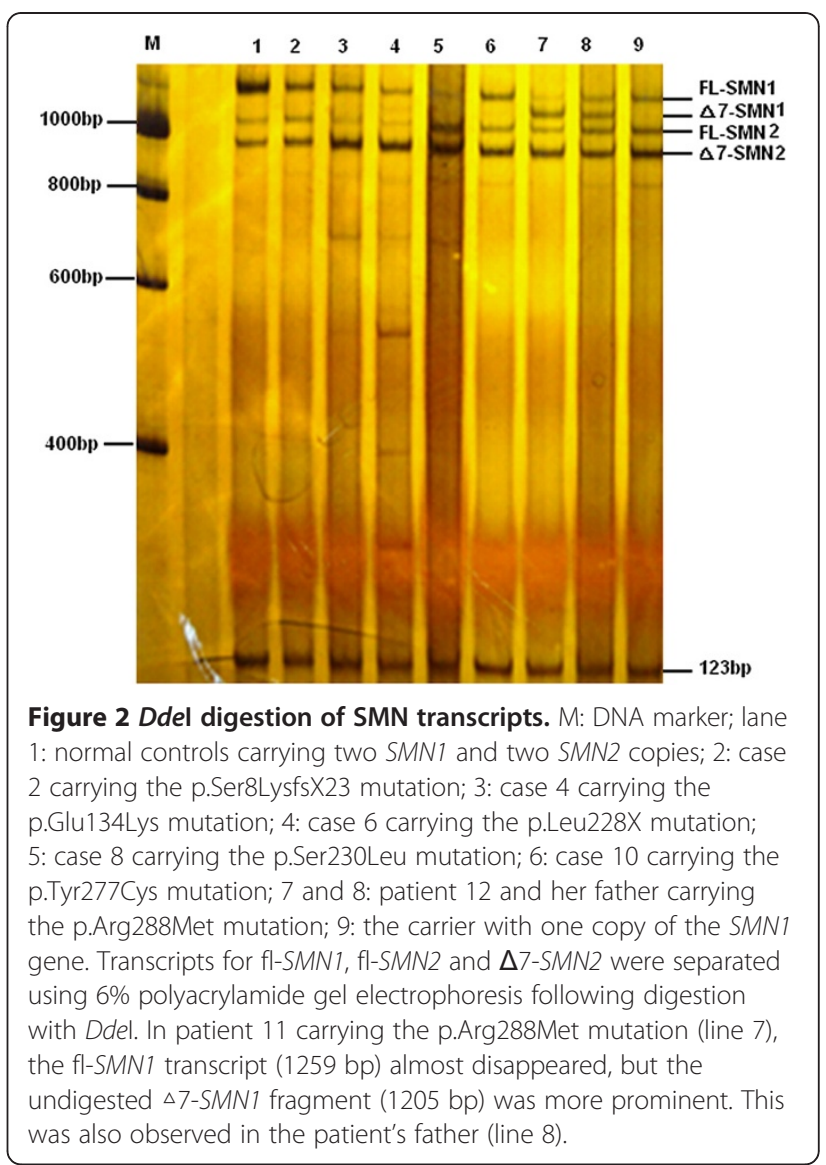

\section{SMN1 subtle mutation}

Six subtle mutations were identified in the present study (Table 1). Five mutations (p.Ser8LysfsX23, p.Leu228X, p.Ser230Leu, p.Glu134Lys, and p.Arg288Met) were detected in more than one patient. A novel mutation (p.Tyr277Cys) located in exon 6 of SMN1 was identified for the first time (Figure 1A). The p.Arg288Met mutation has been previously described [22]. No subtle mutations were detected in two of the patients carrying one copy of SMN1.

\section{Screening for the p.Tyr277Cys mutation}

AS-PCR was performed to screen for the novel p.Tyr277Cys mutation in control individuals (Figure 1B). This mutation was not observed in the 150 control individuals. Sequence alignment of six different species showed that the Tyr277 residue was highly conserved (Figure 1C).

SMN transcripts analysis of patients with subtle mutations A DdeI digest of $S M N$ transcripts assay was carried out to qualitative analysis the $S M N$ transcript in all the patients with subtle mutations. Following DdeI digestion, three obvious products (fl-SMN1, fl-SMN2 and $\triangle 7-S M N 2)$ were detected, without obviously truncated 

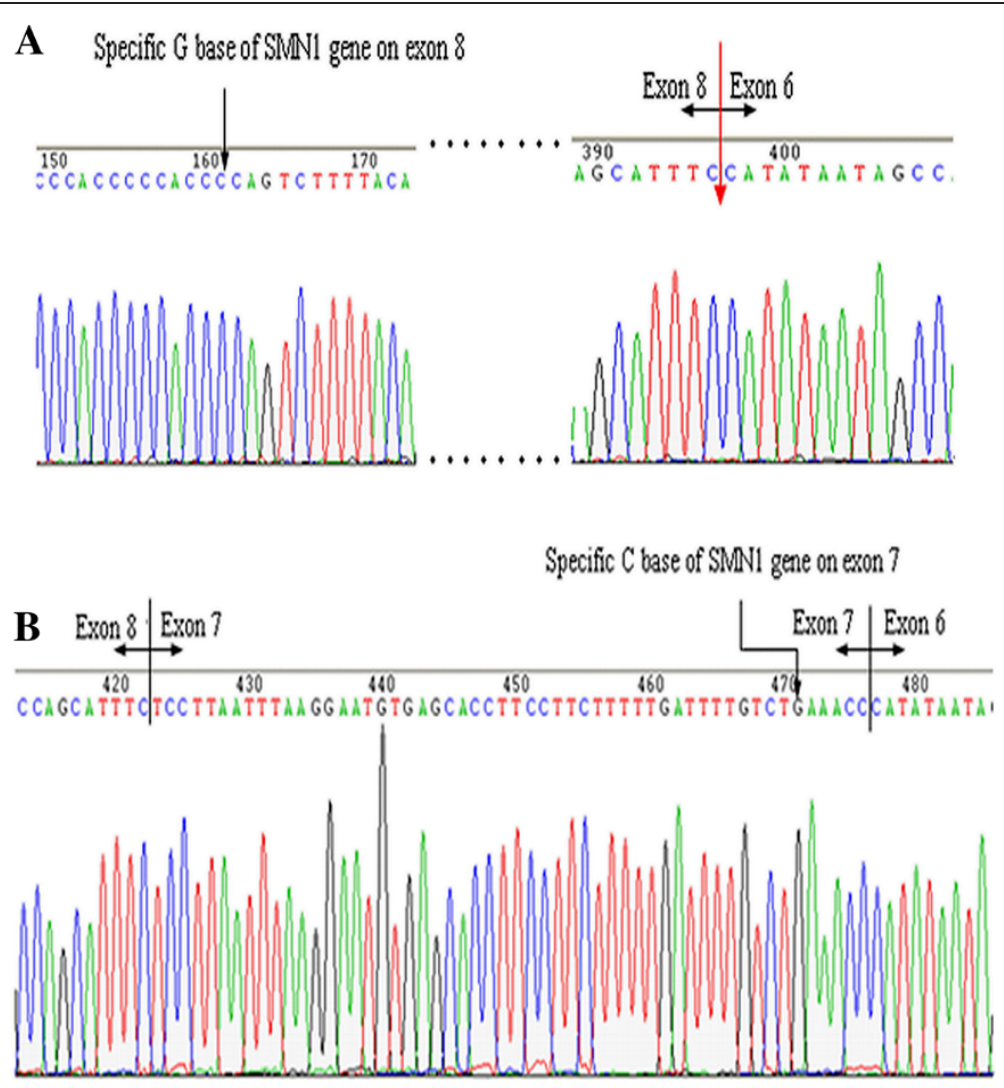

Figure 3 Partial reverse-sequencing map of SMN1 clones for the patient with the p.Arg288Met mutation. (A) Antisense sequencing map of SMN1 clones. The $\mathrm{C}$ base ( $\mathrm{G}$ in the sense sequence) is indicated by the black arrow, which is specific for SMN1 on exon 8 . The red arrow indicates the position of the entire exon 7 sequence that was deleted in the SMN1 clone. (B) Antisense sequencing map of a normal individual. The $\mathrm{G}$ base ( $\mathrm{C}$ in the sense sequence) is indicated by the black arrow and is specific for SMN1 on exon 7 . The intact sequence of exon 7 can be seen between exons 6 and 8 .

or prolonged transcripts in most patients (Figure 2). While in patients carrying the p.Arg288Met mutation, the fl-SMN1 transcript (1259 bp) was rare, but the undigested $\triangle 7-S M N 1$ fragment (1205 bp) was more prominent (Figure 2). An undigested $\triangle 7-S M N 1$ fragment was also observed in their father with the p.Arg288Met mutation.

\section{Clone sequencing of the patients with p.Arg288Met mutation}

The SMN cDNA (from exon 1 to exon 8) of patients with p.Arg288Met were amplified and subclone to the pGEM-T vector. After screening the SMN1 clones and sequencing, we found that all 5 subclones of SMN1 from these patients had lost the entire sequence of exon 7 (Figure 3), while three full-length $S M N$ clones belong to the sequences of $S M N 2$. This result was consistent to the result of DdeI digest of $S M N$ transcripts assay. The p.Arg288Met mutation produces a transcript of $\Delta 7$ SMN1 other than the transcript of fl-SMN1, it implies that it might cause the skip of exon 7 of SMN1 gene.

\section{Comparison of fl-SMN1 transcripts}

To evaluate the effect of subtle mutations on fl-SMN1 levels, we compared fl-SMN1 levels in patients with that of normal controls, and with healthy carriers (Table 3 and Figure 4). The fl-SMN1 levels in normal controls and healthy carriers were $23.77 \pm 7.74$ and $9.47 \pm 5.39$, respectively. The difference between these two groups was statistically significant $(\mathrm{t}=5.296, P=0.000)$. The fl-SMN1 transcript levels in all patients were significantly decreased compared with normal controls $(\mathrm{t}=$ $7.839, P=0.000)$. Compared with healthy carriers carrying one copy of SMN1, patients with the p.Glu134Lys or p.Ser230Leu mutation showed no significant difference (t=1.769, $P=0.094$ and $\mathrm{t}=1.660, P=0.115$, respectively), patients with the novel p.Tyr277Cys mutation presented a slight decrease in fl-SMN1 transcript levels $(\mathrm{t}=2.337, P=0.032)$, while the patients with the p.Ser8LysfsX23, p.Leu228X, and p.Arg288Met mutations were significantly reduced ( $\mathrm{t}$ test, $P=0.000$ ). Especially for the patients carrying p.Arg288Met, fl-SMN1 transcript levels were almost undetectable $(0.017 \pm 0.15)$. 
Table 3 FI-SMN1 transcript levels of controls, carrier, and patients with SMN1 subtle mutations

\begin{tabular}{|c|c|c|c|c|c|c|c|c|c|}
\hline & & Control & Carrier & p.Ser8LysfsX23 & p.Glu134Lys & p.Leu228X & p.Ser230Leu & p.Tyr277Cys & p.Arg288Met \\
\hline$n$ & & 4 & 8 & 2 & 2 & 1 & 2 & 1 & 2 \\
\hline Type & & & & $\|$ & $\|$ & I & $\|\| \|$, & $\|$ & $1, \|$ \\
\hline \multirow[t]{2}{*}{$\mathrm{fl}-\mathrm{SMN} 1$} & Mean \pm SD & $26.49 \pm 13.88$ & $185.71 \pm 148.00$ & $0.61 \pm 0.30$ & $6.30 \pm 2.02$ & $1.57 \pm 0.38$ & $3.54 \pm 0.95$ & $7.19 \pm 2.72$ & $0.05 \pm 0.10$ \\
\hline & Min-Max & $11.16-50.8$ & $29-470$ & $0.22-0.95$ & $3.58-8.26$ & $1.24-1.98$ & $2.94-4.64$ & $4.90-10.20$ & $0-0.19$ \\
\hline \multirow[t]{2}{*}{ GAPDH } & Mean \pm SD & $2322.2 \pm 1174.9$ & $4857.3 \pm 2452.5$ & $3720 \pm 1969$ & $2742 \pm 829.4$ & $3247 \pm 1079$ & $1695 \pm 456$ & $8173 \pm 2957$ & $3002 \pm 1801$ \\
\hline & Min-Max & $1232-4700$ & $1976-8660$ & $2000-6500$ & $1788-3680$ & $2040-4120$ & $1388-2220$ & 5340-11240 & $1315-5300$ \\
\hline \multirow[t]{2}{*}{ corrected fl -SMN1 } & Mean \pm SD & $23.77 \pm 7.74$ & $9.47 \pm 5.39$ & $0.52 \pm 0.46$ & $4.59 \pm 0.68$ & $1.04 \pm 0.39$ & $4.18 \pm 0.20$ & $2.01 \pm 0.89$ & $0.07 \pm 0.15$ \\
\hline & Min-Max & $15.92-34.74$ & $1.05-18.73$ & $0.15-1.15$ & $3.98-5.29$ & $0.69-1.46$ & $3.98-4.38$ & $1.23-2.98$ & $0-0.29$ \\
\hline$P$ value (t-test) & & - & $0.000^{a}$ & $0.000^{b}$ & $0.094^{b}$ & $0.000^{b}$ & $0.11^{\mathrm{b}}$ & $0.032^{b}$ & $0.000^{b}$ \\
\hline
\end{tabular}

$\mathrm{fl}-S M N 1, G A P D H$, and corrected fl- SMN1 indicate transcript levels, measured as no. of molecules per nanogram of total RNA. Superscripted a means that the t-test was carried out between controls and carriers: Superscripted $\mathrm{b}$ means the $\mathrm{t}$-test was performed between carrier and the patients with subtle mutations. 


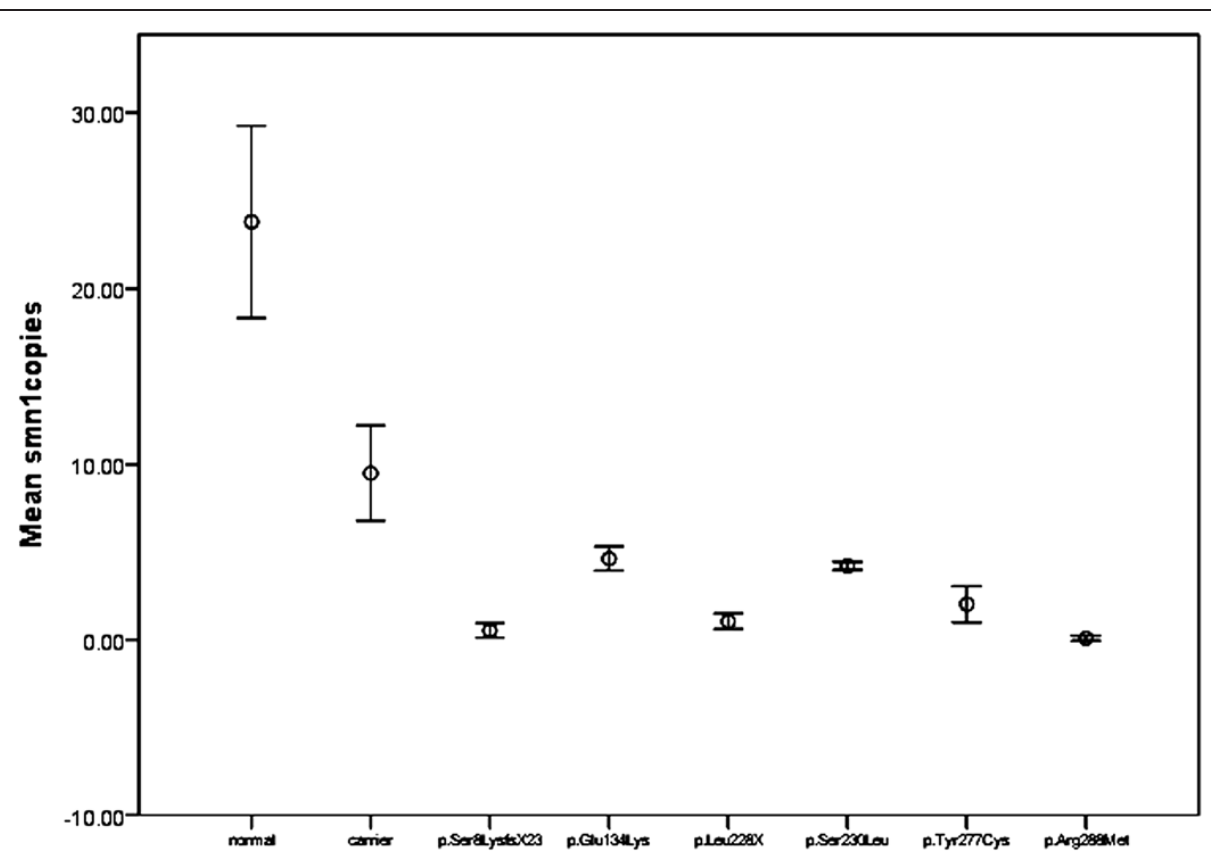

Figure 4 Levels of fl-SMN1 transcripts in controls, carriers and patients with subtle mutations. The dark horizontal lines indicate the $95 \%$ confidence interval. The empty circle indicate the median. Fl-SMN1 transcript levels were significantly different between normal controls and healthy carriers. The levels of transcripts in patients were significantly reduced compared with those in controls. Fl-SMN1 transcript levels in patients with the p.Arg288Met mutation were almost undetectable. In patients with p.Ser8LysfSX23 or p.Leu228X mutations, transcript levels were severely reduced. In the patient carrying the novel p.Tyr277Cys mutation, transcript levels were decreased, while patients with missense mutations (p.Ser230Leu and p.Glu134Lys) showed no significantly decrease compared with carriers.

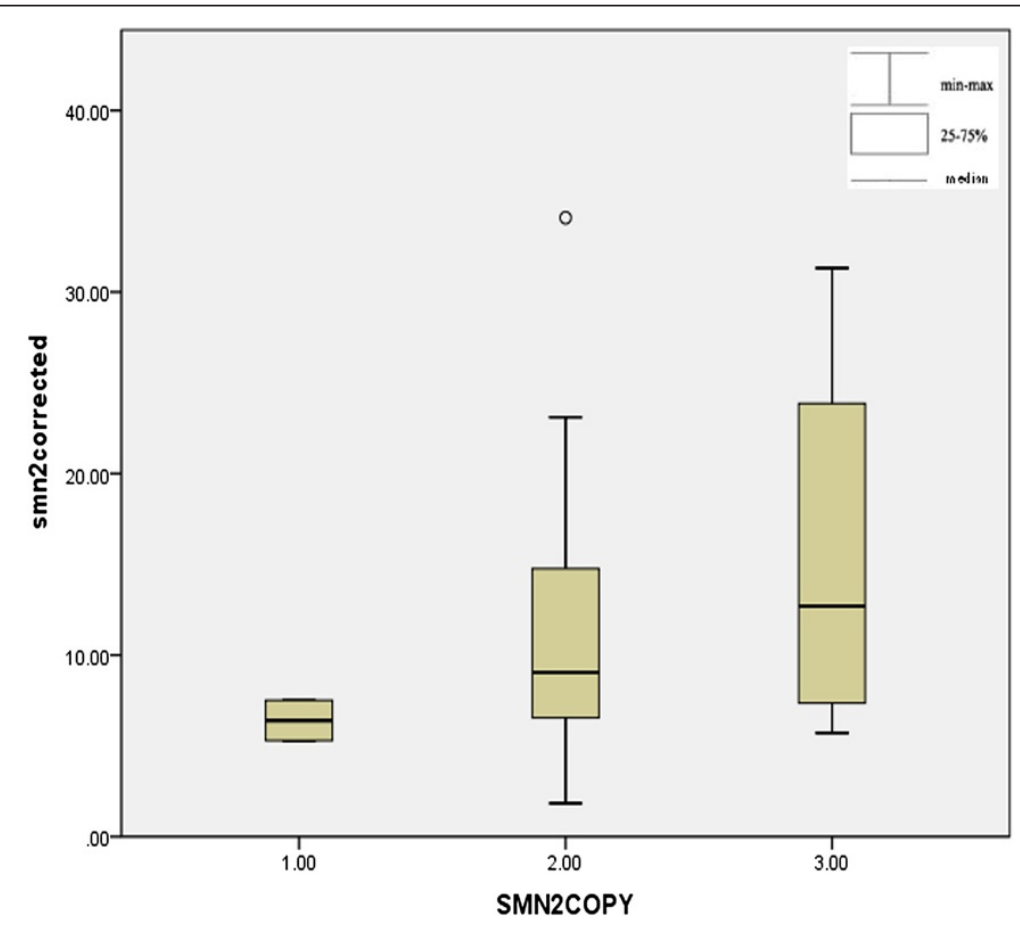

Figure 5 fl-SMN2 transcript levels among the individuals with different SMN2 copy numbers. Mean fl-SMN2 transcript levels in healthy individuals (normal controls and healthy carriers) with a single copy of SMN2 were $6.3895 \pm 1.56,11.48 \pm 8.01$ for those with two copies of SMN2, and $15.61 \pm 11.39$ for patients with three SMN2 copies. No correlation was observed between fl-SMN2 transcript level and SMN2 copy numbers $(F=0.391, P=0.679)$. 
Table 4 Subtle mutations of SMN1 gene identified in Chinese SMA patients

\begin{tabular}{|c|c|c|c|c|c|c|}
\hline Exon/ intron & cDNA mutation & Protein prediction & Mutation type & References & Number of families & Phenotype \\
\hline 5NTR & c. $-39 A>G$ & - & - & Wang CC et al. [21] & 1 & NA \\
\hline \multirow[t]{4}{*}{ Exon 1} & c.22dupA & p.Ser8LysfsX23 & Frameshift & Tsai et al. [18] (first report) & 1 & 1 \\
\hline & & & & Zeng J et al. [23] & 1 & I \\
\hline & & & & Wang CC et al. [21] & $2^{*}$ & NA \\
\hline & & & & This work & 3 & I、॥ \\
\hline Exon 2 & c.84 C > T & p.Ser28Ser & Silence & Wang CC et al. [21] & 1 & NA \\
\hline Exon 3 & $c .400 \mathrm{G}>\mathrm{A}$ & p.Glu134Lys & Missense & This work & 2 & $\|$ \\
\hline \multirow[t]{3}{*}{ Exon 5} & c.683 T > A & p.Leu228X & Nonsense & Tsai et al. [18] (first report) & 1 & 1 \\
\hline & & & & Zeng J et al. [23] & 1 & 1 \\
\hline & & & & This work & 2 & I、II \\
\hline \multirow[t]{2}{*}{ Exon 5} & c. $689 \mathrm{C}>\mathrm{T}$ & p.Ser230Leu & Missense & Zeng J et al. [19] (first report) & 1 & 1 \\
\hline & & & & This work & $1^{*}$ & II、III \\
\hline Exon 6 & $c .830 A>G$ & p.Tyr277Cys & Missense & This work (first report) & 1 & $\|$ \\
\hline Intron 6 & c.835-1 G>A & - & Splice site & Zhu SY et al. [20] & 1 & 1 \\
\hline Exon 7 & $c .863 \mathrm{G}>\mathrm{T}$ & p.Arg288Met & Missense & Qu YJ et al. [22] & 2 & I、II \\
\hline
\end{tabular}

*, indicate these families have two patients who were siblings. $N A$, not available; mutations in bold are common mutations in Chinese SMA patients.

Transcript levels of fl-SMN in different clinical cases

Although there was no correlation between fl-SMN2 transcript level and SMN2 copy numbers (one-way ANOVA, $F=0.391, P=0.679$ ) in normal controls and healthy carriers, the fl-SMN2 transcript level was increased along with SMN2 copy numbers (Figure 5). The fl-SMN2 transcript levels in patients are presented in Table 1, with no significant differences $(\mathrm{F}=1.029, \mathrm{P}=$ 0.430). Total fl-SMN transcript levels (fl-SMN1 + fl$S M N 2)$ were used to assess the correlation between the fl-SMN levels and the clinical severity. The fl-SMN transcript levels in normal controls and healthy carriers were $34.4 \pm 7.1$ and $21.7 \pm 12.7$, respectively, with a significant difference $(\mathrm{t}=2.596, \mathrm{P}=0.017)$. The $\mathrm{fl}-S M N$ transcript levels in all patients were significantly decreased compared with normal controls $(\mathrm{t}=7.060, P=0.000)$. Mean fl-SMN transcript levels in type I $(n=2)$ and type II $(\mathrm{n}=8)$ were $11.27 \pm 6.8$ and $11.67 \pm 6.34$, respectively. For type III $(n=2)$ patients, a slight increase in levels could be observed $(20.00 \pm 9.43)$. Because the number of types I and III patients were low, statistical analysis of fl-SMN transcript levels was only carried out between type II patients and healthy carriers, with a significant difference observed $(t=3.046, P=0.009)$.

\section{Discussion}

Including the results from this study, 61 SMN1 subtle mutations have been detected among diverse populations worldwide. Nine subtle mutations have been identified in Chinese SMA patients [18-23] (Table 4). Among these, the c.1-39A > G, p.Ser8LysfsX23, p.Ser28Ser, p.Leu228X, p.Tyr277Cys, and c.835-1 G > A mutations were only found in Chinese SMA patients, p.Arg288Met mutation had been reported in another East Asian country-Korea [17], only the two mutation (p.Glu134Lys [27], and p.Ser230Leu [28]) were found in Caucasian population. In East Asian country, there was still another mutation p.Trp92Ser only reported in Japanese SMA patient [15]. These reports showed the kinds of SMN1subtle mutation in the Chinese even East Asian population are distinct from those in Caucasian populations. In addition, the type of common mutations differed between Chinese and Caucasian populations. In Chinese SMA patients, p.Ser8LysfsX23 and p.Leu228X seem to be the common mutations, which were detected in seven families $(33.3 \%, 7 / 21)$ and four families $(19.0 \%$, $4 / 21$ ), respectively. While in Caucasian populations, Mutations p.Arg133fsX15, p.Gly261LeufsX8, p.Tyr272Cys and p.Thr274Ile were commonly detected [4,6-8,10, 11,16,25,29-31]. Our research implies that the SMN1 subtle mutations show high heterogeneity in various populations.

Our results show that the level of fl-SMN1 transcripts in normal controls was significantly higher than in healthy carriers, which corresponded to the different number of SMN1 copies carried in these individuals. Although the patients with subtle mutation carried only one SMN1 copy, the effect of these mutations on the flSMN1 transcript levels were different. Based on the qPCR analysis, fl-SMN1 transcript levels in patients with p.Ser8LysfsX23 or p.Leu228X mutations were much lower than in healthy carriers. We presumed the reason for this might be that these two premature termination mutations initiate nonsense-mediated mRNA decay 
(NMD). NMD procedure can result in rapid degradation of SMN1 mRNA [32]. A lack of any significant difference between carriers and patients with missense mutations (p.Glu134Lys and p.Ser230Leu) implied that these missense mutations do not affect transcription of SMN1, or degradation of SMN1 mRNA. However, all the patients with subtle mutations significantly reduced fl-SMN transcript levels compared with normal controls. These results were similar to those seen in patients with a homozygous deletion of SMN1 [26]. We have attempted to assess the correlation between the fl-SMN levels and the clinical severity, while the difference of fl-SMN transcript levels in these patients were not significant. A direct relationship could not be observed between fl-SMN transcript levels and phenotypic severity in this study.

The p.Arg288Met mutation was firstly reported in 2009 [17], and was also found in two unrelated Chinese patients in a previous study [23]. Kang et al. predicted that this mutation was likely to be deleterious to protein structure and function [17]. In our study, an interesting finding was that the SMN1 transcript carrying the p.Arg288Met mutation skipped exon 7 entirely. According to sequence analysis and restriction digestion assays, these two patients with p.Arg288Met mutation produce a transcript corresponding to $\triangle 7-S M N 1$ other than a transcript of fl-SMN1. Subsequent qPCR analysis verified that fl-SMN1 transcripts were almost undetectable $(0.017 \pm 0.15)$. The effect of this mutation was similar to that seen with the $\mathrm{C}$ to $\mathrm{T}$ conversion in SMN2 exon 7 producing $\triangle 7-S M N$ mRNA. This transcript encodes a truncated SMN protein that fails to undergo selfoligomerization of SMN, is unstable, and degrades easily. The pathogenic effect in a patient who has only one copy of SMN1 with the p.Arg288Met mutation is the same as for the homozygous deletion of SMN1. The phenotypes in patients carrying the p.Arg288Met mutation were type I SMA in the two patients and type II SMA in another patient. These results were consistent with a phenotype in SMA patients that have a homozygous deletion of SMN1.

Rare missense mutations were reported to affect the splicing of SMN1. There were two variations that occurred in exon 7 of SMN2 that were described to affect the splicing of $S M N 2$. The $\mathrm{C} \rightarrow \mathrm{T}$ conversion at position six of SMN2 exon 7 was found to cause the entire exon to be skipped [33,34]. The missense mutation p.Gly287Arg (c.859 G > C) in SMN2, recently described as a positive disease modifier, could improve $S M N 2$ exon 7 inclusion [35]. Many studies have revealed that multiple cis-elements and splicing factors participate in the regulation of SMN exon 7 splicing. Singh et al. [36] showed that the Conserved tract is a positive element located in the middle of exon 7 at positions 16-44. Hofmann et al. [37] revealed binding of exonic splicing enhancers (ESEs) with Htra2-b1 in the middle of exon 7 at positions 19-27. In this study, our results show that the p.Arg288Met mutation may influence splicing of exon 7 , causing the entire exon to be skipped. Serine/argininerich protein-binding ESE elements were not observed in wild-type or mutant sequences at c.863 using ESE finder 2.0 (http://rulai.cshl.edu/tools/ESE2). However, the p.Arg288Met mutation at position 29 of exon 7 was located within the Conserved tract element, and near where the ESE binds with Htra2-b1. We speculate that an unascertained ESE, regulating SMN exon 7 splicing, might exist in the Conserved tract element and that this variant of $\mathrm{G}>\mathrm{T}$ in c.863 may influence the ESE site or produce a new exonic splicing silencer element (ESS).

The tyrosine/glycine-rich sequence (Y/G box), containing highly conserved residues Tyr268 and Gly279 (YXXGYXXGYXXG) in the C-terminus of SMN, is an essential self-oligomerization domain of SMN. It plays role in assembling of the SMN complex and participating in pre-mRNA splicing [38,39]. The aromatic amino acids Tyr268, Tyr272, and Tyr276, each at three-base intervals, seem to be more important than other amino acids in the $\mathrm{Y} / \mathrm{G}$ box because two SMN monomers might form a stable dimer through aromatic stacking of these tyrosines [16]. When these three tyrosines mutate, SMN self-oligomerization could be severely disturbed. To date, several missense mutations in the Y/G box, including p.Tyr272Cys, p.His273Arg, p.Thr274Ile, p.Gly275Ser, p.Gly279Cys and p.Gly279Val, have been reported $[4,7,10-14,16,40-44]$. Among these, p.Tyr272Cys and p.Gly279Val were reported to be usually associated with the more severe type I form of SMA [4,11-13,44]. The p.Thr274Ile, p.Gly275Ser and p.Gly279Cys mutations were generally associated with the milder, type II and III phenotypes [7,10-13,16,41-43].

In this paper, the p.Tyr277Cys mutation within the Y/G box is reported for the first time. Although Tyr277 is an aromatic amino acid, it is near the critical Tyr276 residue. Both Tyr276 residues from two SMN monomers require adequate space to form aromatic stacking. We speculate that Tyr277 replaced by a Cys residue might disturb the space structure of aromatic stacking, thereby affecting the stability of the SMN dimer. The patient carrying the p.Tyr277Cys mutation also had two copies of SMN2. The mean fl-SMN transcript level in this patient was $6.54 \pm 4.33$, which was much lower than that in normal controls $(34.4 \pm 7.1)$ and healthy carriers (21.7 \pm 12.7). The phenotype of this patient in our study was indicative of type II SMA. Although Tyr277 is a highly conserved residue, we predicted that the p.Tyr277Cys mutation might be a milder mutation affecting the structure of the Y/G box.

It was worth to note that two siblings (cases 8 and 9) with the same p.Ser230Leu mutation displayed different 
phenotypes. The elder brother was diagnosed as type II SMA, while his younger sister was type III SMA. Cases 8 and 9 carried two SMN2 copies, with mean fl-SMN transcript levels of $12.22 \pm 7.07$ and $14.83 \pm 4.45$, respectively. The difference between them was not significant $(\mathrm{t}=-0.453, P=0.682)$. We hypothesized that there might be other modifying factors that affect phenotypes, such as Plastin 3, which has been reported as a sex-specific protective modifier of SMA [45-47].

\section{Conclusion}

In conclusion, six SMN1 subtle mutations were identified in 12 patients, including the novel p.Tyr277Cys mutation. Based on our research and other studies, p.Ser8LysfsX23 and p.Leu228X mutations seem to be the common SMN1 subtle mutations in Chinese patients. This preliminary study revealed that SMN1 subtle mutations in Chinese SMA were different to those observed in Caucasian populations. A qRT-PCR assay implied that p.Ser8LysfsX23, p.Leu228X, and p.Arg288Met mutations affected fl-SMN1 transcript levels. Besides, we discovered that the p.Arg288Met mutation disturbed the splicing of exon7 SMN1 pre-mRNA, resulting in SMN1 transcripts skipping exon 7.

\section{Competing interests}

The authors declare no potential competing interests with respect to the authorship and/or publication of this article.

\section{Authors' contributions}

SF conceived of, designed and organized the study, contributed to obtaining the funding, and helped to critically revise the manuscript. QY contributed to obtaining the funding, carried out the experiments, analyzed data, and wrote the initial draft of the manuscript. DJ helped to carry out the subtle mutation analysis experiments (Cloning and sequencing); LE was responsible for diagnosis and management of patients; $\mathrm{BJ}$ contributed to the writing of the manuscript; JY and WH contributed to the sequencing experiments. All authors approved the final version of the manuscript submitted for publication.

\section{Acknowledgments}

We are grateful to the patients and their families for their participation and cooperation in providing blood samples and information. We greatly acknowledge Professors Wang Li-wen (Children's Hospital Affiliated Capital Institute of Pediatrics), Xiong Hui and Yang Yan-ling (First Hospital of Peking University), and Zou Li-ping (PLA General Hospital) for their clinical support. The research was supported by the National Natural Science Foundation of China (Project Nos. 81050034 and 81100933), the Natural Science Foundation of Beijing Municipal (Project No. 7112020),the Capital Health Research and Development of Special(Project No. 2011-1008-03) and the Science Foundation of the Capital of Institute of Pediatrics (Project No. 11-A05).

\section{Author details}

'Department of Medical Genetics, Capital Institute of Pediatrics, Beijing, China. ${ }^{2}$ Department of Neurology, Children's Hospital Affiliated Capital Institute of Pediatrics, Beijing, China.

Received: 9 March 2012 Accepted: 14 September 2012

Published: 20 September 2012

\section{References}

1. Pearn J: Incidence, prevalence, and gene frequency studies of chronic childhood spinal muscular atrophy. J Med Genet 1978, 15:409-413.
2. Ogino S, Wilson RB, Gold B: New insights on the evolution of the SMN1 and SMN2 region: simulation and meta-analysis for allele and haplotype frequency calculations. Eur J Hum Genet 2004, 12:1015-1023.

3. Zerrres K, Davies KE: 59th ENMC International Workshop: Spinal Muscular Atrophies: recent progress and revised diagnostic criteria 17-19 April 1998, Soestduinen, The Netherlands. Neuromusc Disord 1999, 9:272-278.

4. Lefebvre $S$, Bürglen $L$, Reboullet $S$, Clermont $O$, Burlet $P$, Viollet $L$, Benichou B, Cruaud C, Millasseau P, Zeviani M, Paslier DL, Frezal J, Cohen D, Weissenbach J, Munnich A, Melki J: Identification and characterization of a spinal muscular atrophy-determining gene. Cell 1995, 80:155-165.

5. Lorson CL, Androphy EJ: An exonic enhancer is required for inclusion of an essential exon in the SMA-determining gene SMN. Hum Mol Genet 2000, 9:259-265.

6. Bussaglia $E$, Clermont $O$, Tizzano E, Lefebvre S, Bürglen L, Cruaud $C$, Urtizberea JA, Colomer J, Munnich A, Baiget M, Melki J: A frame-shift deletion in the survival motor neuron gene in Spanish spinal muscular atrophy patients. Nat Genet 1995, 11:335-337.

7. Hahnen E, Schönling J, Rudnik-Schöneborn S, Raschke H, Zerres K, Wirth B: Missense mutations in exon 6 of the survival motor neuron gene in patients with spinal muscular atrophy (SMA). Hum Mol Genet 1997, 6:821-825.

8. Parsons DW, MCAndrew PE, Monani UR, Mendell JR, Burghes AHM, Prior TW: An 11 base pair duplication in exon 6 of the SMN gene produces a type I spinal muscular atrophy (SMA) phenotype: further evidence for the SMN as the primary SMA-determining gene. Hum Mol Genet 1996 , 5:1727-1732.

9. Brahe C, Clermont O, Zappata S, Tiziano F, Melki J, Neri G: Frameshift mutation in the survival motor neuron gene in a severe case of SMA type I. Hum Mol Genet 1996, 5:1971-1976.

10. Parsons DW, McAndrews PE, lannaccone ST, Mendell JR, Burghes AH, Prior TW: Intragenic telSMN mutations: frequency, distribution, evidence of a founder effect, and modification of the spinal muscular atrophy phenotype by cenSMN copy number. Am J Hum Genet 1998, 63:1712-1723.

11. Wirth B, Herz M, Wetter A, Moskau S, Hahnen E, Rudnik-Schöneborn S, Wienker T, Zerres K: Quantitative analysis of survival motor neuron copies: identification of subtle SMN1 mutations in patients with spinal muscular atrophy, genotype-phenotype correlation, and implications for genetic counseling. Am J Hum Genet 1999, 6:1340-1356.

12. Clermont $O$, Burlet $P$, Benit $P$, Chanterau $D$, Saugier-Veber $P$, Munnich $A$, Cusin V: Molecular analysis of SMA patients without homozygous SMN1 deletions using a new strategy for identification of SMN1 subtle mutations. Hum Mutat 2004, 24:417-427.

13. Sun Y, Grimmler M, Schwarzer V, Schoenen F, Fischer U, Wirth B: Molecular and Functional Analysis of Intragenic SMN1 Mutations in Patients with Spinal Muscular Atrophy. Hum Mutat 2005, 25:64-71.

14. Zapletalová E, Hedvicáková P, Kozák L, Vondrácek P, Gaillyová R, Maríková T, Kalina Z, Jüttnerová V, Fajkus J, Fajkusová L: Analysis of point mutations in the SMN1 gene in SMA patients bearing a single SMN1 copy. Neuromuscul Disor 2007, 17:476-481.

15. Kotani T, Sutomo R, Sasongko TH, Sadewa AH, Gunadi, Minato T, Fujii E, Endo S, Lee MJ, Ayaki H, Harada Y, Matsuo M, Nishio H: A novel mutation at the N-terminal of SMN Tudor domain inhibits its interaction with target proteins. J Neurol 2007, 254:624-630.

16. Alías $L$, Bernal $S$, Fuentes-Prior $P$, Barceló MJ, Also E, Martínez-Hernández R, Rodríguez-Alvarez FJ, Martín Y, Aller E, Grau E, Peciña A, Antiñolo G, Galán E, Rosa AL, Fernández-Burriel M, Borrego S, Millán JM, Hernández-Chico C, Baiget M, Tizzano EF: Mutation update of spinal muscular atrophy in Spain: molecular characterization of 745 unrelated patients and identification of four novel mutations in the SMN1 gene. Hum Genet 2009, 125:29-39.

17. Kang SH, Cho SI, Chae JH, Chung KN, Ra EK, Kim SY, Seong MW, Kim JY, Park SS: False homozygous deletions of SMN1 exon 7 using Dral PCRRFLP caused by a novel mutation in spinal muscular atrophy. Genet Test Mol Biomarkers 2009, 13:511-513.

18. Tsai CH, Jong YJ, Hu CJ, Chen CM, Shih MC, Chang CP, Chang JG: Molecular analysis of SMN, NAIP and P44 genes of SMA patients and their families. J Neurol Sci 2001, 190:35-40.

19. Zeng J, Lin YH, Yan AZ, Cai MY, Ke LF, Lan FH: Mutation analysis of SMN gene in a patient and his family with spinal muscular atrophy. Chin J Med Genet (Chin) 2009, 26:139-143. 
20. Sheng-Yuan Z, Xiong F, Chen YJ, Yan TZ, Zeng J, Li L, Zhang YN, Chen WQ, Bao XH, Zhang C, Xu XM: Molecular characterization of SMN copy number derived from carrier screening and from core families with SMA in a Chinese population. Eur J Hum Genet 2010, 18:978-984.

21. Wang CC, Chang JG, Chen YL, Jong YJ, Wu SM: Multi-exon genotyping of SMN gene in spinal muscular atrophy by universal fluorescent PCR and capillary electrophoresis. Electrophoresis 2010, 31:2396-2404.

22. Qu YJ, Song F, Yang YL, Jin YW, Bai JL: Compound heterozygous mutation in two unrelated cases of Chinese spinal muscular atrophy patients. Chin Med J 2011, 134:385-389.

23. Zeng J, Lin Y, Yan A, Ke L, Zhu Z, Lan F: Establishment of a molecular diagnostic system for spinal muscular atrophy experience from a clinical laboratory in China. J Mol Diag 2011, 13:41-47.

24. Vezain M, Gérard B, Drunat S, Funalot B, Fehrenbach S, N'guyen-Viet V, Vallat JM, Frébourg T, Tosi M, Martins A, Saugier-Veber P: A leaky splicing mutation affecting SMN1 exon 7 inclusion explains an unexpected mild case of spinal muscular atrophy. Hum Mutat 2011, 32:989-994.

25. van der Steege G, Grootscholten PM, van der Vlies P, Draaijers TG, Osinga J, Cobben JM, Scheffer H, Buys CH: PCR-based DNA test to confirm clinical diagnosis of autosomal recessive spinal muscular atrophy. Lancet 1995, 345:985-986.

26. Tiziano FD, Pinto AM, Fiori S, Lomastro R, Messina S, Bruno C, Pini A, Pane M, D'Amico A, Ghezzo A, Bertini E, Mercuri E, Neri G, Brahe C: SMN transcript levels in leukocytes of SMA patients determined by absolute real-time PCR. Eur J Hum Genet 2010, 18(1):52-58.

27. Clermont O, Burlet P, Cruaud C, Bertrandy S, Melki J, Munnich A, Lefebvre S: Mutation analysis of the SMN gene in undeleted SMA patients. Am J Hum Genet 1997, 61:A329.

28. Nölle A, Zeug A, van Bergeijk J, Tönges L, Gerhard R, Brinkmann H, Al Rayes S, Hensel N, Schill Y, Apkhazava D, Jablonka S, O'mer J, Srivastav RK, Baasner A, Lingor P, Wirth B, Ponimaskin E, Niedenthal R, Grothe C, Claus P: The spinal muscular atrophy disease protein SMN is linked to the rho-kinase pathway via profilin. Hum Mol Genet 2011, 20(24):4865-4878

29. Martín Y, Valero A, del Castillo E, Pascual SI, Hernández-Chico C: Genetic study of SMA patients without homozygous SMN1 deletions: identification of compound heterozygotes and characterisation of novel intragenic SMN1 mutations. Hum Genet 2002, 110:257-263.

30. Cuscó I, López E, Soler-Botija C, Barceló MJ, Baiget M, Tizzano EF: A genetic and phenotypic analysis in Spanish spinal muscular atrophy patients with c.399_402del AGAG, the most frequently found subtle mutation in the SMN1 gene. Hum Mutat 2003, 22:136-143.

31. Parsons DW, MCAndrew PE, Allinson PS, Parker WD Jr, Burghes AH, Prior TW: Diagnosis of spinal muscular atrophy in an SMN non-deletion patient using a quantitative PCR screen and mutation analysis. J Med Genet 1998, 35:674-676.

32. Brichta L, Garbes L, Jedrzejowska M, Grellscheid SN, Holker I, Zimmermann K, Wirth B: Nonsense-mediated messenger RNA decay of survival motor neuron 1 causes spinal muscular atrophy. Hum Genet 2008, 123:141-153.

33. Cartegni L, Hastings ML, Calarco JA, de Stanchina E, Krainer AR: Determinants of exon 7 splicing in the spinal muscular atrophy genes, SMN1 and SMN2. Am J Hum Genet 2006, 78:63-77.

34. Kashima T, Manley JL: A negative element in SMN2 exon 7 inhibits splicing in spinal muscular atrophy. Nat Genet 2003, 34:460-463.

35. Vezain M, Saugier-Veber P, Goina E, Touraine R, Manel V, Toutain A, Fehrenbach S, Frébourg T, Pagani F, Tosi M, Martins A: A rare SMN2 variant in a previously unrecognized composite splicing regulatory element induces exon 7 inclusion and reduces the clinical severity of spinal muscular atrophy. Hum Mutat 2010, 31:E1110-E1125.

36. Singh NN, Androphy EJ, Singh RN: In vivo selection reveals combinatorial controls that define a critical exon in the spinal muscular atrophy genes. RNA 2004, 10:1291-1305.

37. Hofmann Y, Lorson CL, Stamm S, Androphy EJ, Wirth B: Htra2-beta 1 stimulates an exonic splicing enhancer and can restore full-length SMN expression to survival motor neuron 2 (SMN2). Proc Natl Acad Sci U S A 2000, 97:9618-9623.

38. Lorson CL, Strasswimmer J, Yao JM, Baleja JD, Hahnen E, Wirth B, Le T, Burghes AH, Androphy EJ: SMN oligomerization defect correlates with spinal muscular atrophy severity. Nat Genet 1998, 19:63-66.

39. Wang J, Dreyfuss G: Characterization of functional domains of the SMN protein in vivo. J Biol Chem 2001, 276:45387-45393.
40. Prior TW: Spinal muscular atrophy diagnostics. J Child Neurol 2007, 22:952-956.

41. Bürglen L, Patel S, Dubowitz V, Melki J, Muntoni F: A novel point mutation in the SMN gene in a patient with type III spinal muscular atrophy. In: First Congress of the World Muscle Society. Amsterdam: Elsevier; 1996:S39.

42. Skordis LA, Dunckley MG, Burglen L, Campbell L, Talbot K, Patel S, Melki J, Davies KE, Dubowitz V, Muntoni F: Characterization of novel point mutations in the survival motor neuron gene SMN, in three patients with SMA. Hum Genet 2001, 108:356-357.

43. Talbot K, Ponting CP, Theodosiou AM, Rodrigues NR, Surtees R, Mountford $R$, Davies KE: Missense mutation clustering in the survival motor neuron gene: a role for a conserved tyrosine and glycine rich region of the protein in RNA metabolism? Hum Mol Genet 1997, 6:497-500.

44. Wang $\mathrm{CH}$, Papendick BD, Bruinsma P, Day JK: Identification of a novel missense mutation of the SMN (T) gene in two siblings with spinal muscular atrophy. Neurogenetics 1998, 1:273-276.

45. Oprea GE, Kröber S, McWhorter ML, Rossoll W, Müller S, Krawczak M, Bassell GJ, Beattie CE, Wirth B: Plastin 3 Is a Protective Modifier of Autosomal Recessive Spinal Muscular Atrophy. Science 2008, 320:524-527.

46. Stratigopoulos G, Lanzano P, Deng L, Guo J, Kaufmann P, Darras B, Finkel R Tawil R, McDermott MP, Martens W, Devivo DC, Chung WK: Association of plastin 3 expression with disease severity in spinal muscular atrophy only in postpubertal females. Arch Neurol 2010, 67:1252-1256.

47. Bernal S, Also-Rallo E, Martínez-Hernández R, Alías L, Rodríguez-Alvarez FJ, Millán JM, Hernández-Chico C, Baiget M, Tizzano EF: Plastin 3 expression in discordant spinal muscular atrophy (SMA) siblings. Neuromuscul Disord 2011, 21:413-419.

doi:10.1186/1471-2350-13-86

Cite this article as: Yu-jin et al: Subtle mutations in the SMN1 gene in Chinese patients with SMA: p.Arg288Met mutation causing SMN1 transcript exclusion of exon7. BMC Medical Genetics 2012 13:86.

\section{Submit your next manuscript to BioMed Central and take full advantage of:}

- Convenient online submission

- Thorough peer review

- No space constraints or color figure charges

- Immediate publication on acceptance

- Inclusion in PubMed, CAS, Scopus and Google Scholar

- Research which is freely available for redistribution

Submit your manuscript at www.biomedcentral.com/submit
C) Biomed Central 